\title{
Special issue on surveillance systems for air and airport traffic control
}

\author{
GASPARE GALATI AND PIET VAN GENDEREN
}

Increasing safety and efficiency levels in air transport requires modern control and traffic management (ATM) systems for aircraft in air and in ground operations, as well as for service vehicles on the airport surface. The related Communications, Navigation and Surveillance infrastructures call for enhanced positioning and identification techniques such as multilateration (MLAT) and wide Area MLAT (WAM), automatic dependent surveillance-broadcast (ADS-B), as well as automatic location and management of service vehicles in the airport. This enhanced surveillance infrastructure is spatially distributed (i.e. with many receiving or transmitting/ receiving stations) and logically distributed (i.e. with local and central processing and with fusion of different information sources, including the traditional primary and secondary radar). The present and future satellite navigation systems are (or will be) a backbone for some of them.

In this frame, new system architectures and new algorithms for integrity monitoring and for multi-sensor data fusion are required.

Security and defence systems use similar algorithms for passively locating targets based on measurements of time of arrival (TOA) and its differences (TDOA) as well as of Doppler frequency and its differences (FDOA), possibly combined with angular/direction measurements (AOA/DOA).

The workshop "Enhanced Surveillance for Vehicles and Aircraft", held in Capri on September 2008 (ESAV'08), addressed the various topics highlighted above. Not only did engineering and scientific staff contribute, but also policy makers from Europe and the United States of America presented their point of view on the ongoing programs and future needs. It appears that the automatic dependent surveillance, i.e. the cooperative positioning and identification of aircraft based on on-board derived data (such as the GPS ones) and communicated toward other aircraft and ground-based stations, is gaining relevance. Conversely, it is also clear that the independent surveillance (based on radar, either "primary", i.e. requiring no cooperation at all from the target, or "secondary", i.e. the secondary surveillance radar -(SSR) based on today's standard and mandatory mode S transponder on-board) will continue to be relevant for monitoring both air and surface traffic. There is general consensus that the stringent surveillance requirements can only be satisfied by the "fusion" of different sensors, including the

Corresponding author:

Piet van Genderen

Email: P.vanGenderen@tudelft.nl independent and the non-cooperative ones, the latter being essential for security reasons, too.

The 10 papers we have selected for this special issue provide a snapshot of the work underway in the domains of the various surveillance systems and useful techniques for air traffic control (ATC). The rigorous approach chosen in the ATC domain to assess and verify the performance of these systems is also shown.

Papers in this special issue address:

- novel methods dealing with the processing of signals in the highly congested SSR frequency band,

- "dual use" non-cooperative classification of some specific classes of objects,

- time- and frequency difference of arrival passive location techniques,

- surface movement detection and identification,

- trajectory reconstruction techniques to assess the performance of ATC surveillance.

We trust that this special issue provides a true flavor of the current developments and trends, and therefore serves the best interests of the engineering and scientific communities.

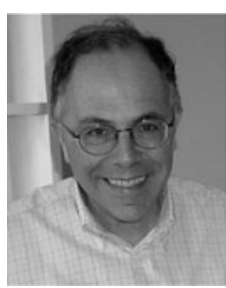

Gaspare Galati is a full professor of Radar Theory and Techniques at the Tor Vergata University of Rome. He received the Dr. Ing. degree (Laurea) at the University of Rome "La Sapienza" and started his professional career in the company Selenia S.p.A in 1970. His main interests are in radar theory and techniques, detection and estimation, navigation and air traffic management. His present research activity is mostly related to new surveillance systems for air traffic control and to multifunction radar.

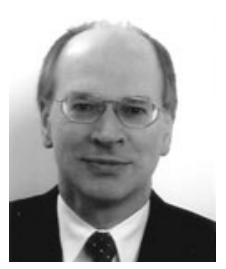

Piet van Genderen graduated in 1971 from the University of Twente in Enschede, The Netherlands in information theory. He joined the National Aerospace Laboratory (NLR) in Amsterdam in 1971, working on radar and processing for air traffic control. In 1979 he moved to Hollandse Signaalapparaten, now Thales. Since 1994 he also holds a chair on radar system design at the Delft University of Technology in The Netherlands. His current research interests are in the design of wide band, agile radar, in particular in the design of waveforms and algorithms for extracting object features from the received video. 